\title{
Tangence
}

\section{Le schéma tensif}

Synthèse et propositions

\section{Tensive Modelization}

\section{Synthesis and Propositions}

\section{Louis Hébert}

Numéro 79, automne 2005

Art et avatars de la conversation

URI : https://id.erudit.org/iderudit/012854ar

DOI : https://doi.org/10.7202/012854ar

Aller au sommaire du numéro

Éditeur(s)

Tangence

ISSN

1189-4563 (imprimé)

1710-0305 (numérique)

Découvrir la revue

Citer cet article

Hébert, L. (2005). Le schéma tensif : synthèse et propositions. Tangence, (79), 111-139. https://doi.org/10.7202/012854ar
Résumé de l'article

Dispositif de la sémiotique post-greimassienne, le schéma tensif a été introduit par Jacques Fontanille et Claude Zilberberg. L'auteur entend montrer le potentiel opératoire de ce dispositif, en s'écartant au besoin de son cadre d'origine. Prenant en compte les trajets orthogonaux, l'auteur propose notamment de faire passer de quatre à douze les trajets que l'on peut effectuer sur un schéma tensif à sectorisation dyadique ; pour les trajets sur un schéma tensif à sectorisation triadique, il suggère de s'inspirer de la typologie des courbes d'euphorie esthétique qu'il a dressée. Une application à des pensées de Pascal montre la nécessité de ces compléments théoriques tout en présentant un dernier ajout : la prise en compte des points de vue. 


\title{
Le schéma tensif. Synthèse et propositions \\ Louis Hébert, Université du Québec à Rimouski
}

\begin{abstract}
Dispositif de la sémiotique post-greimassienne, le schéma tensif a été introduit par Jacques Fontanille et Claude Zilberberg. L'auteur entend montrer le potentiel opératoire de ce dispositif, en s'écartant au besoin de son cadre d'origine. Prenant en compte les trajets orthogonaux, l'auteur propose notamment de faire passer de quatre à douze les trajets que l'on peut effectuer sur un schéma tensif à sectorisation dyadique; pour les trajets sur un schéma tensif à sectorisation triadique, il suggère de s'inspirer de la typologie des courbes d'euphorie esthétique qu'il a dressée. Une application à des pensées de Pascal montre la nécessité de ces compléments théoriques tout en présentant un dernier ajout: la prise en compte des points de vue.
\end{abstract}

Dispositif de la sémiotique post-greimassienne, le schéma tensif a été introduit par Jacques Fontanille et Claude Zilberberg ${ }^{1}$. Nous entendons, à travers une présentation théorique puis une application à des pensées de Pascal, montrer le potentiel opératoire de ce dispositif, en nous écartant au besoin de son cadre d'origine.

\section{Théorie}

\subsection{Postulats théoriques}

Il n'y a pas lieu de discuter de tous les postulats théoriques associés au schéma tensif. Nous intéresse principalement ici son caractère opératoire brut, si l'on peut dire. Cependant, pour mémoire, voici les plus importants de ces postulats:

1. Dans ce chapitre, nous nous inspirons librement de Jacques Fontanille (Sémiotique du discours, Limoges, Presses de l'Université de Limoges, 1993, p. 69-77 et 109-116) et de Claude Zilberberg («Précis de grammaire tensive», Tangence, Rimouski/Trois-Rivières, $\mathrm{n}^{\circ}$ 70, automne 2002, p. 111-143). 
1. Intensité et extensité constituent, respectivement, le plan du contenu (plan des signifiés) et le plan de l'expression (plan des signifiants $)^{2}$. Puisque tout signe provient de la réunion de ces deux plans, tout signe, en principe du moins, est descriptible en termes tensifs ${ }^{3}$.

2. L'intensité est de l'ordre du sensible (c'est-à-dire du perceptible et/ou du ressenti affectif, nous y reviendrons); l'extensité, de l'intelligible ${ }^{4}$.

3. L'intensité renvoie aux états d'âme (passions); l'extensité, aux états de choses ${ }^{5}$.

4. L'intensité touche l'intéroceptivité (la sensibilité dont les stimulus proviennent de l'organisme même); l'extensité, l'extéroceptivité (la sensibilité dont les stimulus proviennent du monde externe) ${ }^{6}$.

5. L'intensité correspond à la visée; l'extensité, à la saisie 7 . La visée et la saisie sont définies comme suit: "cette tension en direction du monde [...] est l'affaire de la visée intentionnelle; la position, l'étendue et la quantité caractérisent en revanche les limites et les propriétés du domaine de pertinence, c'està-dire celles de la saisie. La présence engage donc les deux opérations élémentaires [...] : la visée, plus ou moins intense, et la saisie, plus ou moins étendue ${ }^{8} »$.

6. L'intensité correspond à l'«affection» (aux passions); l'extensité, à la cognition ${ }^{9}$.

7. L'intensité régit, contrôle l'extensité ${ }^{10}$.

2. Jacques Fontanille, Sémiotique du discours, ouvr. cité, p. 72.

3. Chez Claude Zilberberg ("Précis de grammaire tensive», art. cité, p. 115), il semble que tout signe, tout signe discursif du moins, est descriptible en termes tensifs: "toute grandeur discursive se trouve qualifiée au titre de l'intensité et de l'extensité».

4. Claude Zilberberg, "Précis de grammaire tensive», art. cité, p. 115; Jacques Fontanille, Sémiotique du discours, ouvr. cité, p. 110.

5. Voir Claude Zilberberg, "Précis de grammaire tensive», art. cité, p. 115: «la tensivité est le lieu imaginaire où l'intensité, c'est-à-dire les états d'âme, le sensible, et l'extensité, c'est-à-dire les états de choses, l'intelligible, se joignent les uns les autres".

6. Jacques Fontanille, Sémiotique du discours, ouvr. cité, p. 72.

7. Jacques Fontanille, Sémiotique du discours, ouvr. cité, p. 73 et 98.

8. Jacques Fontanille, Sémiotique du discours, ouvr. cité, p. 39.

9. Jacques Fontanille, Sémiotique du discours, ouvr. cité, p. 110.

10. Claude Zilberberg, "Précis de grammaire tensive», art. cité, p. 114, 115 et 116. 
8. Intensité et extensité se décomposent chacune en deux sousdimensions: ce sont, respectivement, le tempo et la tonicité, la temporalité et la spatialité ${ }^{11}$.

9. Les deux opérations fondamentales de l'intensité sont l'augmentation et la diminution; celles de l'extensité sont le tri (qui augmente la diversité et/ou le nombre) et le mélange (qui diminue la diversité et/ou le nombre) ${ }^{12}$.

10. L'implication relève de l'intensité et la concession de l'extensité $^{13}$.

11. Le schéma tensif relève d'une sémiotique du continu (et complète la sémiotique du discontinu ${ }^{14}$ ), d'une sémiotique de l'intervalle (et complète la sémiotique des relations entre termes ${ }^{15}$ ), d'une sémiotique de l'événement (et complète la sémiotique des états ${ }^{16}$ ).

\section{Remarque: homologations des postulats}

Les postulats que nous venons de rappeler s'inscrivent pour la plupart dans une série d'homologations, c'est-à-dire de relations où le premier et le second termes d'une opposition sont corrélés, respectivement, au premier et au second termes des autres oppositions participant de l'homologation. Dressons une liste de ces oppositions homologuées: signifié/ signifiant, plan des contenus/plan de l'expression, perceptible (sensible)/intelligible, interne/externe, visée/saisie, passion/ cognition, états d'âme/états de choses, intéroceptif/extéroceptif. Dans la citation qui suit, Fontanille établit plusieurs de ces homologations: "l'intensité caractérise le domaine interne, intéroceptif, et qui deviendra le plan du contenu; l'étendue caractérise le domaine externe, extéroceptif, et qui deviendra le plan de l'expression; la corrélation entre les deux domaines résulte de la prise de position d'un corps propre, celui-là même qui est le siège de l'effet de présence sensible; elle est donc proprioceptive $^{17} »$.

11. Claude Zilberberg, "Précis de grammaire tensive», art. cité, p. 116.

12. Claude Zilberberg, Éléments de sémiotique tensive, Québec, Presses de l’Université Laval (à paraître).

13. Claude Zilberberg, Éléments de sémiotique tensive, ouvr. cité.

14. Claude Zilberberg, "Précis de grammaire tensive», art. cité, p. 112.

15. Claude Zilberberg, "Précis de grammaire tensive», art. cité, p. 125.

16. Claude Zilberberg, "Précis de grammaire tensive", art. cité, p. 139-141.

17. Jacques Fontanille, Sémiotique du discours, ouvr. cité, p. 72. 
Le postulat qui touche au sensible/intelligible pose problème. Chez Zilberberg et Fontanille, le mot sensible doit-il être pris au sens habituel de "perceptible» pour entrer dans l'opposition courante entre ce qui passe par les sens, se perçoit et ce qui uniquement se conçoit; ou doit-il être pris au sens passionnel (quand, par exemple, on dit d'une personne qu'elle est «sensible» ou qu'elle «ressent» telle émotion) pour entrer dans l'opposition entre le cour et la raison ${ }^{18}$ ? Chez Fontanille, les deux acceptions du mot sensible semblent cohabiter : "la substance est sensible - perçue, sentie, pressentie - , la forme est intelligible - comprise, signifiante ${ }^{19}$ », écrit-il, et plus loin le sensible est explicitement associé à l'affect ${ }^{20}$.

Partant de là, notons que si, globalement, la perception serait de l'ordre de l'intensité, l'une de ses formes particulières, l'extéroceptivité, est rapportée à l'extensité. Ce principe du classement global d'un élément dans l'un des termes d'une opposition et du classement local des variétés de ce même élément dans chacun des deux termes de cette opposition est courant. Par exemple, dans un autre secteur de la sémiotique greimassienne, on trouve la structure suivante: les signifiés discursifs, comme tout signifié, relèvent de l'intelligible, mais certains évoquent des perceptions, les signifiés figuratifs (par exemple, celui du mot "rouge»), d'autres pas, les signifiés thématiques (par exemple, celui du mot «gloire»). En revanche, à notre avis, la corrélation du perceptible, globalement, à l'intensité et de l'intelligible à l'extensité crée un problème de cohérence dans les homologations. En effet, si signifiant et signifié sont tous deux choses mentales, il faut admettre que les signifiants (par exemple un phonème, étudié en phonologie) ont des corrélats directs dans le monde physique (par exemple, un son particulier réalisant un phonème, son étudié en phonétique) et, à cet égard, tendent vers le perceptible. On pourrait objecter que la distinction signifiant/signifié est toute relationnelle, et qu'un plan de l'expression peut devenir le plan du contenu d'une autre sémiotique et un plan du contenu, devenir le plan d'expression d'une

18. Usant de cette opposition, Blaise Pascal, dans les Pensées, écrit: "Guerre intestine de l'homme entre la raison et les passions. " Voir ses Euvres complètes, tome II, édition présentée, établie et annotée par Michel Le Guern, Paris, Gallimard, coll. «Bibliothèque de la Pléiade», 2000, p. 771. Dans cette édition, les Pensées (p. 543-900) rassemblent 782 fragments; la citation faite ici est tirée du fragment 528.

19. Jacques Fontanille, Sémiotique du discours, ouvr. cité, p. 40.

20. Jacques Fontanille, Sémiotique du discours, ouvr. cité, p. 110. 
autre sémiotique... Il n'en demeure pas moins que les signifiants typiques procèdent du perceptible. Ajoutons d'autres arguments. L'opposition états d'âme/états de choses semble s'homologuer naturellement à intelligible/perceptible : l'âme est traditionnellement associée à l'imperceptible et opposée en cela au corps et, plus généralement, aux éléments matériels. Enfin, la temporalité et la spatialité, présentées comme sous-dimensions de l'extensité, sont les propriétés des éléments perceptibles plus que des éléments intelligibles. L'emploi métaphorique n'est évidemment pas à exclure, et l'on peut, par exemple, parler de l'«étendue» de la connaissance même si elle ne porte que sur des objets abstraits; mais c'est l'emploi littéral qui devrait décrire les matériaux typiques de l'extensité.

\subsection{Valeurs et valences}

Dans le schéma tensif, une valeur donnée est constituée par la combinaison de deux «valences» (ou dimensions), l'intensité et l'extensité (ou étendue ${ }^{21}$ ). L'extensité est l'étendue à laquelle s'applique l'intensité; elle correspond à la quantité, à la variété, à l'étendue spatiale ou temporelle des phénomènes ${ }^{22}$. Les deux

21. En logique, on oppose l'intension d'un concept ou sa compréhension, c'est-àdire l'ensemble des traits de sens qui le définissent (par exemple, un homme est un mammifère, bipède, etc.), à son extension, c'est-à-dire l'ensemble des phénomènes qu'il recouvre (par exemple, l'ensemble des hommes). Si l'extensité entretient des similitudes sémantiques fortes avec l'extension, il n'en va pas de même, comme on peut le voir, pour l'intensité et l'intension.

22. Selon Zilberberg, («Précis de grammaire tensive», art. cité, p. 116), tempo et tonicité constituent les deux sous-dimensions de l'intensité, tandis que la temporalité et la spatialité sont les deux sous-dimensions de l'extensité; les fonctifs de chacune des deux dimensions, c'est-à-dire les éléments qui les constituent, sont, pour l'intensité, faible/éclatant et, pour l'extensité, concentré/diffus (voir également Claude Zilberberg, Éléments de sémiotique tensive, ouvr. cité). Il nous semble que la temporalité et la spatialité laissent un résidu dans ce que devrait recouvrir l'extensité. En effet, l'extensité touche, en plus de l'étendue spatiale ou temporelle, la quantité et la variété des phénomènes en cause; or, ceux-ci ne sont pas toujours réductibles au spatial, sauf à prendre le terme dans un sens métaphorique, et peuvent correspondre, par exemple, à des concepts, comme dans le cas de l' "étendue» de la connaissance. Par ailleurs, lorsqu'on qualifie l'étendue de diffuse ou de concentrée (voir, par exemple, Jacques Fontanille, Sémiotique du discours, ouvr. cité, p. 46), donnant par là une indication de densité, c'est qu'on combine implicitement deux aspects de l'étendue, à savoir la quantité des phénomènes et leur étendue spatiale ou temporelle. Fontanille (Sémiotique du discours, ouvr. cité, p. 110) donne l'intensité et l'affect, avec un «etc.», comme éléments 
valences relèvent du quantitatif: la première, du mesurable; la seconde, du nombrable ${ }^{23}$.

\subsection{Force des valences}

Intensité et extensité connaissent chacune des variations dans leur force, sur une échelle continue allant de la force nulle à la force maximale, voire infinie. Comme d'autres dispositifs (le carré sémiotique, le carré véridictoire, le modèle actantiel, etc.), le schéma tensif est à la fois un réseau, une structure conceptuelle et une représentation visuelle de cette structure ${ }^{24}$. Si on place l'intensité sur l'ordonnée d'un plan et l'extensité sur son abscisse, on obtient alors une représentation visuelle à deux axes.

pouvant figurer dans l'axe des intensités. La question se pose: peut-on placer sur un axe une valence composite? Si la réponse est oui, on peut dès lors distinguer entre une utilisation analytique du schéma tensif et une utilisation synthétique. L'utilisation synthétique s'autorise à placer sur l'un des axes une valence composée: ainsi, on reportera sur l'axe des intensités l'intensité d'un élément autre que celui qui se trouve sur l'axe de l'extensité: par exemple, l'intensité de l'évaluation positive affectée à la connaissance (valence composée, tandis que l'intensité appliquée à la connaissance serait une valence simple) pourra augmenter en fonction de l'étendue de la connaissance (valence simple).

23. Claude Zilberberg (Éléments de sémiotique tensive, ouvr. cité) écrit à propos de l'unité à décrire tensivement: "l'unité doit être mesurable et/ou dénombrable, mesurable en intensité, dénombrable en extensité». Plus loin, il dit croire que la sémiotique tensive permet «la réconciliation de la qualité et de la quantité, ce qui est le propre du concept de mesure, lequel permet, à moindres frais, la qualification des quantités et la quantification des qualités ».

24. Si le dispositif tensif est qualifié de schéma, ce n'est pas tant en raison de la représentation visuelle schématique qui lui est associée que du sens technique de "schématisme», terme qui, «dans la tradition issue de Kant, désigne la médiation entre le concept et l'image, et, plus généralement, entre les catégories de l'entendement et les phénomènes sensibles " (Jacques Fontanille, Sémiotique du discours, ouvr. cité, p. 110). En effet, l'un des postulats théoriques qui accompagne le schéma tensif est celui de la médiation de l'intelligible (l'intensité) et du sensible (l'extensité) qui s'opère en lui. 
Les deux axes du schéma tensif

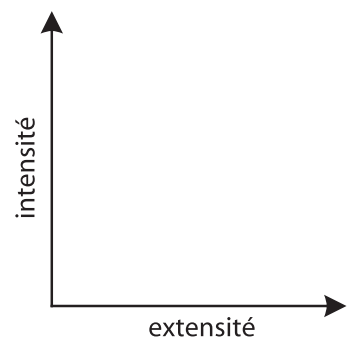

Un phénomène donné occupera, en fonction de la force de l'intensité et de l'extensité qui le caractérisent, une ou plusieurs positions données sur ce plan ${ }^{25}$. Il est également possible de donner une représentation du schéma sous forme de tableau (nous en donnerons un exemple plus bas).

\subsection{Secteurs des valences et zones du schéma tensif}

Il est possible de distinguer différents secteurs, en nombre variés, sur les échelles tensives.

\subsubsection{Sectorisation dyadique}

Par exemple, si l'on distingue pour chaque valence deux secteurs, un secteur de force basse (ou secteur atone) et un secteur de force élevée (ou secteur tonique), on obtient quatre combinaisons possibles entre les valences, définissant autant de zones ${ }^{26}$ :

25. Des phénomènes, comme la courbe de la tragédie française dont nous parlerons plus loin, nécessitent la combinaison de plusieurs schémas pour être représentés.

26. Claude Zilberberg, quant à lui (Éléments de sémiotique tensive, ouvr. cité), opte pour une partition en deux aires, délimitée par la bissectrice du plan. Audessus de la bissectrice figure l'aire des valeurs d'absolu et, au bas de celle-ci, l'aire des valeurs d'univers: "Ce qui importe dès qu'on se met à l'écoute des discours, ce n'est pas la signification en soi des deux ordres, mais ce que chacun représente "aux yeux" de l'autre: (1) selon les valeurs d'univers, sensibles aux valences extensives, les valeurs d'absolu sont certes intenses, mais présentent le grave défaut d'être concentrées [extensité faible]; les valeurs d'univers elles-mêmes sont faibles [en intensité], mais présentent l'avantage, 
Zone 1 : intensité basse et extensité basse

Zone 2 : intensité élevée et extensité basse

Zone 3 : intensité basse et extensité élevée

Zone 4 : intensité élevée et extensité élevée

En représentation schématique, cela donnera:

Les quatre zones du schéma tensif

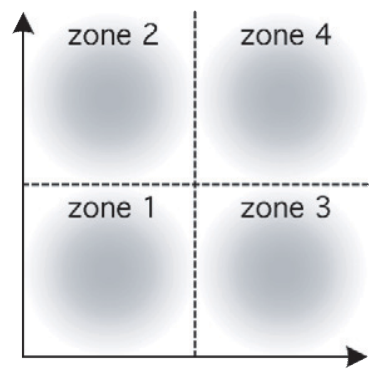

extensité

Soit un groupe d'émotions que nous appellerons l'attachement aux êtres. Au sein de ce groupe, nous distinguerons les émotions uniquement en termes quantitatifs, tout en étant conscient qu'une approche qualitative pourrait considérer que, par exemple, entre amour et amitié, il y une différence de nature et non pas (seulement) de quantité. L'axe des intensités se rapporte à l'intensité de l'émotion et l'axe des étendues au nombre d'êtres qui sont l'objet de cette émotion de la part d'un sujet donné. En prenant en compte une partition en quatre zones, on distinguera quatre grands types d'émotion. Dans la zone un, nous placerons (a) l'amour (ordinaire); dans la zone deux, (b) le "grand amour» ou l'«l'amour d'une vie»; dans la zone trois, (c) l'amitié, et dans la zone quatre, (d) l'«amour universel» ou la compassion. Raffinons

à leurs yeux supérieures d'être diffuses [extensité forte]; (2) selon les valeurs d'absolu, sensibles surtout aux valences intensives, les valeurs d'univers sont diffuses, mais faibles; les valeurs d'absolu elles-mêmes sont certes concentrées, mais leur éclat [forte intensité] compense infiniment ce défaut. Ainsi chaque ordre de valeurs disqualifie nécessairement son vis-à-vis en vertu des préférences valencielles qu'il retient». On remarquera que la portion typique de l'aire des valeurs d'absolu et de celle des valeurs d'univers correspond, respectivement, aux zones deux et trois. 
l'analyse. Distinguons en termes d'extensité, d'une part, l'amour et le grand amour, celui-ci s'appliquant en principe à moins d'êtres que celui-là et, d'autre part, l'amitié et l'amour universel qui, comme son nom l'indique, s'applique en principe à plus d'êtres que le premier. Par ailleurs, distinguons en termes d'intensité, d'une part, l'amour et l'amitié, cette dernière étant un sentiment en principe moins intense et, d'autre part, le grand amour et l'amour universel, pour peu que l'on considère ce dernier comme l'amour absolu en termes d'extensité mais également d'intensité. Visuellement, cette analyse plus fine peut être représentée ainsi:

\section{Configuration tensive des émotions d'attachement}

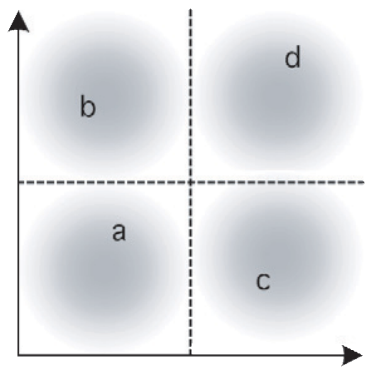

extensité

D'autres distinguos pourraient être faits, par exemple, entre la compassion chrétienne et la compassion bouddhique, laquelle, plus extense, rayonne non seulement vers les humains mais les autres êtres vivants (et tant pis pour les pierres et autres «êtres» inanimés!).

\subsubsection{Sectorisations autres que dyadiques}

Nous avons donné un exemple de sectorisation dyadique, laquelle, lorsqu'elle s'applique sur les deux axes à la fois, génère quatre zones. Cependant, d'autres sectorisations sont possibles. Ainsi, une sectorisation triadique pourra distinguer, sur l'un et/ou l'autre des axes, les forces basse, moyenne et élevée; une sectorisation pentadique, quant à elle, les forces nulle, basse, moyenne, élevée et maximale (voire infinie), par exemple. Une sectorisation tétradique sur les deux axes permettrait d'attribuer une zone spécifique à chacune des quatre émotions auxquelles nous nous sommes attardées; certaines des douze autres zones 
créées par cette sectorisation pourraient convenir à la description d'autres émotions d'attachement. Dans l'application qui se trouve ci-dessous, nous abordons la question de la «traduction» d'un schéma soumis à une sectorisation donnée en un schéma soumis à une autre sectorisation.

\subsection{Aspects dynamiques du schéma tensif}

Abordons maintenant les aspects dynamiques du schéma tensif. Intensité et extensité connaissent deux types de corrélation. La corrélation est dite converse ou directe si, d'une part, l'augmentation de l'une des deux valences s'accompagne de l'augmentation de l'autre et, d'autre part, la diminution de l'une entraîne la diminution de l'autre. Elle est alors de type "plus... plus...» ou «moins... moins...». La corrélation est dite inverse si l'augmentation de l'une des deux valences s'accompagne de la diminution de l'autre et réciproquement. Elle est alors de type «plus... moins...» ou «moins... plus...».

Remarque: schéma tensif et carré sémiotique

Les relations entre le carré sémiotique et le schéma tensif restent à approfondir. Rabattu sur le carré sémiotique, le schéma tensif permet de rendre compte de manière plus précise des variations de la quantité des éléments composant un métaterme, typiquement le terme complexe. Par exemple, il permet de décrire non seulement les termes en relations inverse - ce qu'autorise déjà la notion d'équilibre/dominance - , mais également les termes en relation converse, relation où l'augmentation de la force d'un terme va de pair avec l'augmentation de la force de l'autre terme et, mutatis mutandis, pour ce qui est de la diminution de la force. Dans l'utilisation que nous venons d'en faire, le schéma tensif est généralisé puisque, à la différence du carré sémiotique, le schéma tensif ne nécessite pas que des éléments opposés, car encore faut-il qu'ils relèvent, respectivement, de l'intensité et de l'extensité. 
Exemples de corrélations directe et inverse

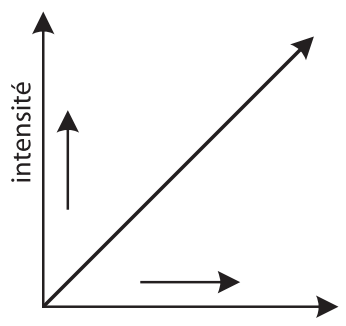

extensité

exemple de corrélation directe

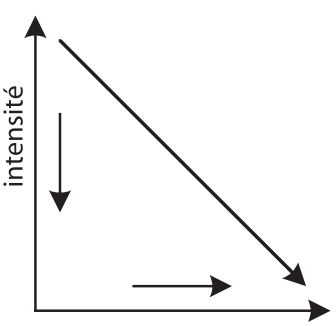

extensité

exemple de corrélation inverse

Les deux corrélations définissent des zones de corrélation qui peuvent être approximativement représentées comme suit:

Zones de corrélation

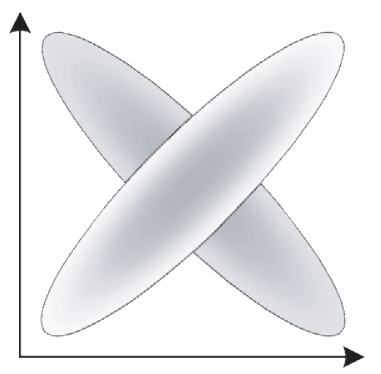

zone de corrélation directe

\section{zone de} corrélation inverse

extensité

Remarque: pertinence des quatre zones

Tous les points du plan sont susceptibles de correspondre à des valeurs du même phénomène. Cependant, deux principes organisateurs se dégagent: d'une part, la différence entre les corrélations directe et inverse détermine deux grandes directions de la variation; d'autre part, «la conjugaison des degrés les plus forts et des degrés les plus faibles sur les deux axes détermine des zones extrêmes. Tous les points de l'espace interne sont pertinents; mais les zones extrêmes de chaque corrélation sont les zones les plus typiques de la catégorie examinée. La combinaison entre ces deux principes permet de 
dégager quatre grandes zones typiques de la catégorie ${ }^{27} »$. Certes, le dyadisme est une sectorisation prégnante; cependant, il nous semble que, malgré leur probabilité moins grande, les sectorisations autres, en particulier la triadique, ne doivent pas être écartées a priori, mais seulement si elles ne sont pas pertinentes pour le phénomène à décrire.

Reprenons notre exemple des émotions d'attachement. L'homme moyen est tributaire, en principe, d'une relation inverse en vertu de laquelle plus une émotion est intense moins elle s'applique à un grand nombre d'êtres. La zone de l'amour universel lui est étrangère.

\subsection{Schémas tensifs élémentaires}

En combinant la nature converse ou inverse de la relation tensive et son orientation dans le temps, on obtient quatre schémas tensifs élémentaires ${ }^{28}$ :

27. Jacques Fontanille, Sémiotique du discours, ouvr. cité, p. 74.

28. Comme on le voit, les tracés tensifs, même lorsque le temps n'est pas en abscisse, ne se prêtent pas pour autant uniquement à des approches atemporelles. En effet, la typologie des quatre schémas tensifs repose sur un critère de succession des positions tensives (toute succession se produisant, en principe du moins, dans le temps). Les schémas tensifs combinent alors trois variables: intensités, extensités et positions temporelles. 
Schémas tensifs élémentaires

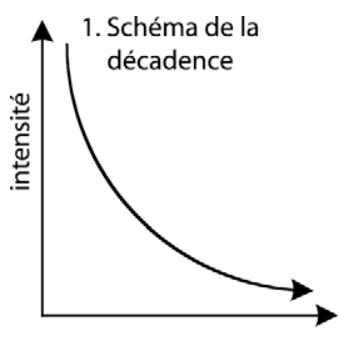

extensité

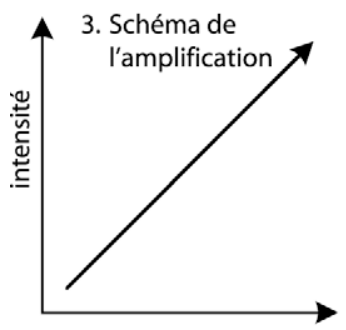

extensité

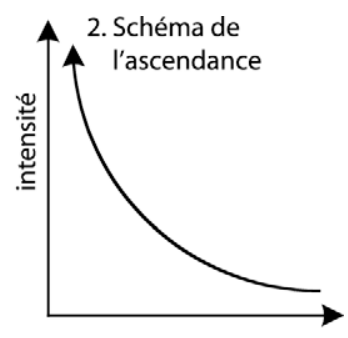

extensité

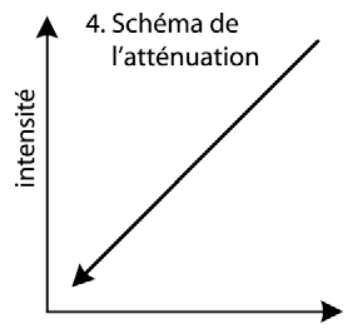

extensité

Remarque: le schéma tensif et la géométrie

Les représentations du schéma tensif s'inspirent de la géométrie mais n'en relèvent pas. Cela explique certaines libertés et variations représentationnelles. Par exemple, pour représenter les corrélations directe et inverse, Zilberberg emploie des ellipses modifiées, en ce qu'elles rejoignent les points zéro de l'ordonnée et de l'abscisse ${ }^{29}$; Fontanille, quant à lui, emploie des courbes elliptiques pour la corrélation inverse et la droite pour la corrélation directe. En principe, les ellipses de Fontanille devraient être modifiées, et il le fait d'ailleurs remarquer:

Dans le cas de la corrélation directe, la direction des variations de positions suit globalement l'orientation de la bissectrice de l'angle; dans le cas de la corrélation inverse, les variations de positions suivent une direction perpendiculaire à cette

29. Claude Zilberberg, «Précis de grammaire tensive», art. cité, p. 118. 
bissectrice, direction qui peut être aussi représentée par un arc dont les deux extrémités rejoignent les deux axes de base ${ }^{30}$.

Cependant, sans doute pour des raisons de lisibilité des schémas, les représentations qu'il donne de relations inverses dans les schémas de la décadence et de l'ascendance ne font pas se rejoindre la courbe et les axes ${ }^{31}$. Quant à nous, pour maintenir la lisibilité des schémas et leur cohérence, nous n'avons pas fait se toucher, comme il se devrait, les courbes ou droites et les axes.

Remarque: effets des schémas tensifs élémentaires

Les schémas tensifs élémentaires se définissent, selon Fontanille, comme des variations d'équilibre entre le sensible (l'intensité, l'affect, etc.) et l'intelligible (le déploiement dans l'étendue, le mesurable, la compréhension ${ }^{32}$ ). Ces variations conduisent «soit à une augmentation de la tension affective, soit à une détente cognitive. L'augmentation de l'intensité apporte la tension; l'augmentation de l'étendue apporte la détente ${ }^{33} »$. En conséquence, (1) le schéma de la décadence procure une détente cognitive; (2) le schéma de l'ascendance, une tension affective; (3) le schéma de l'amplification, une tension affective et cognitive; (4) le schéma de l'atténuation, une détente générale, affective et cognitive ${ }^{34}$.

\subsubsection{Le schéma de la décadence}

Le schéma de la décadence (ou schéma descendant) peut s'observer, par exemple, dans le passage entre ce que les publicitaires appellent l'accroche, fortement affective mais souvent faible en étendue, et le reste de l'affiche ${ }^{35}$.

\subsubsection{Le schéma de l'ascendance}

Le schéma de l'ascendance (ou schéma ascendant) se trouve, par exemple, dans le passage, en littérature, entre le corps d'une nouvelle et sa fin (chute), d'étendue plus faible mais d'intensité

30. Jacques Fontanille, Sémiotique du discours, ouvr. cité, p. 74.

31. Jacques Fontanille, Sémiotique du discours, ouvr. cité, p. 111.

32. Jacques Fontanille, Sémiotique du discours, ouvr. cité, p. 110.

33. Jacques Fontanille, Sémiotique du discours, ouvr. cité, p. 110.

34. Jacques Fontanille, Sémiotique du discours, ouvr. cité, p. 111-112.

35. Jacques Fontanille, Sémiotique du discours, ouvr. cité, p. 112. 
plus forte; le même phénomène se produit, par exemple, entre le corps du sonnet et sa fin (ou pointe) ${ }^{36}$.

\subsubsection{Le schéma de l'amplification}

Le schéma de l'amplification (ou schéma amplifiant) se retrouve, par exemple, dans la plupart des constructions symphoniques qui nous conduisent de la ligne à peine audible, tenue par un seul instrument ou quelques-uns, à sa reprise par de plus en plus d'instruments et ce, avec une intensité croissante ${ }^{37}$. Pensons au Boléro de Ravel.

\subsubsection{Le schéma de l'atténuation}

Le schéma de l'atténuation (ou schéma atténuant) apparaît, par exemple, dans la comédie ou le drame à résolution heureuse où, à la fin, le nombre des problèmes et leur intensité se réduisent, voire s'abolissent.

\subsection{Combinaisons de schémas tensifs élémentaires}

Deux schémas tensifs ou plus peuvent s'enchaîner dans une production sémiotique. Quand cet enchaînement est stéréotypé, on parle de schéma tensif canonique ${ }^{38}$. Par exemple, la tragédie classique française enchaîne, du quatrième au cinquième acte, un schéma d'atténuation (les conflits diminuent en nombre et s'apaisent) et un schéma d'amplification (la catastrophe advient et se généralise).

Remarque: schémas tensifs canoniques

Fontanille nomme «schémas tensifs canoniques ${ }^{39}$ » les schémas tensifs composés "qui conjuguent et enchaînent plusieurs schémas tensifs, sous une forme figée et immédiatement reconnaissable dans une culture donnée ". "Comme ils sont caractéristiques d'un type ou d'un genre, ils guident $a$ priori la compréhension du discours, et ils ont alors le statut

36. Jacques Fontanille, Sémiotique du discours, ouvr. cité, p. 113.

37. Jacques Fontanille, Sémiotique du discours, ouvr. cité, p. 113.

38. Jacques Fontanille, Sémiotique du discours, ouvr. cité, p. 110.

39. Jacques Fontanille, Sémiotique du discours, ouvr. cité, p. 110. 
de schémas culturels, placés sous convention ou hérités de la tradition: c'est la raison pour laquelle on les appelle des schémas canoniques ${ }^{40} »$. Nos combinaisons de schémas tensifs sont plus générales puisqu'elles peuvent, en principe du moins, ne pas être figées, stéréotypées et quand elles le sont, elles peuvent l'être au niveau individuel et non pas nécessairement au niveau de toute une culture.

Il nous semble qu'il y a deux façons de comprendre les schémas tensifs élémentaires en termes d'échelle. La première considère que les schémas tensifs élémentaires proprement dits doivent couvrir l'ensemble des positions prévues sur au moins un des axes. Alors, si l'on considère, pour reprendre l'exemple de la tragédie, que dans l'apaisement l'intensité passe d'élevée à moyenne, le tracé qui représente cet apaisement ne ressortit pas d'un schéma tensif de l'atténuation mais d'une partie seulement de ce schéma. La seconde, que nous ferons nôtre, ne voit là qu'une variation d'échelle d'un même schéma tensif élémentaire intégral. Ainsi, la figure en forme de pointe appelée "cusp ${ }^{41}$ » sera-t-elle considérée, peu importe sa taille, comme constituée de la combinaison de deux schémas tensifs élémentaires, par exemple du schéma de l'amplification suivi du schéma de la décadence.

\subsection{Schémas orthogonaux}

Aucun des schémas tensifs élémentaires ne prévoit que l'une des valences soit constante tandis que l'autre varie, ce qui donnerait des droites orthogonales, horizontales ou verticales. Or, il est possible de prévoir déductivement l'existence de phénomènes dont la description nécessite des tracés impliquant une constante (au besoin, considérons que nous venons d'attester l'existence de tels phénomènes simplement en en évoquant la possibilité...). Qui plus est, nous verrons plus loin, dans notre analyse de la théorie pascalienne de la connaissance, un exemple de tels phénomènes. Combien peut-on prévoir de ces droites orthogonales? Dans une sectorisation dyadique, on trouve deux tracés verticaux, l'un à extensité faible constante, l'autre à extensité forte constante, et deux tracés horizontaux, l'un à intensité faible constante et l'autre

40. Jacques Fontanille, Sémiotique du discours, ouvr. cité, p. 116.

41. Pour un autre exemple d'utilisation d'un cusp, voir Jacques Fontanille, Sémiotique et littérature, Paris, Presses universitaires de France, 1999, p. 155. 
à intensité forte constante. Comme chacune des droites peut être parcourue dans deux directions (par exemple, pour une intensité constante, l'extensité peut-être croissante ou décroissante), on obtient ainsi huit nouveaux trajets, pour un grand total de douze types de trajets.

Puisque des tracés à constante sont, à notre avis, nécessaires sinon avérés, il est indispensable de les intégrer, d'une manière ou d'une autre, dans la description. À cet égard, plusieurs possibilités se présentent. Nous en voyons deux principales:

1. Il faut distinguer schémas tensifs élémentaires et déplacements ${ }^{42}$ tensifs élémentaires. Il s'agit d'une solution similaire à celle que nous avons proposée en ce qui a trait à la succession des positions sur un carré sémiotique ${ }^{43}$. Tout en reconnaissant que les contraintes que l'on fait généralement peser sur l'enchaînement de ces positions sur un carré sémiotique (il faudrait nécessairement passer de l'un des termes à sa négation pour atteindre ensuite le terme contraire) peuvent servir à produire un modèle décrivant la génération d'une catégorie (de deux éléments liés par une relation de contrariété), nous soutenons qu'une production sémiotique peut les enchaîner à sa guise (par exemple, en passant directement d'un terme à son contraire, disons de la beauté à la laideur, sans passer par la non-beauté). Bref, les schémas élémentaires tensifs représentent des modes de génération des valeurs et les déplacements élémentaires, leur succession dans une production sémiotique donnée. Sur les douze types de déplacements, quatre correspondent à un schéma tensif élémentaire.

2. Pour une sectorisation dyadique, il existe quatre schémas tensifs élémentaires et huit schémas non tensifs élémentaires inscriptibles dans un nouveau dispositif qui n'est pas le schéma tensif, même s'il procède, comme lui, de deux axes. Ce dispositif permet de rendre compte de tous les types de combinaisons, tensives ou non, entre deux variables intensives ou quantitatives. On pourra alors représenter les variations de

42. Nous préférons «déplacement» à "parcours», puisque ce dernier terme est associé, dans la sémiotique greimassienne, à une succession d'étapes plus ou moins obligatoires, par exemple dans le "parcours génératif de la signification".

43. Voir Louis Hébert, Dispositifs pour l'analyse des textes et des images, Limoges/ Québec, Presses de l'Université de Limoges/Presses de l'Université Laval (à paraître). 
deux intensités de deux phénomènes distincts: par exemple, celles de l'ambition (en abscisse) et celles de l'amour (en ordonnée) dont est victime un Julien Sorel dans Le rouge et le noir de Stendhal. On pourra aussi représenter les variations de l'étendue de deux phénomènes distincts: par exemple, celles des espèces monétaires (argent liquide) et celles de la monnaie scripturale (billets à échéance, etc.) du Grandet de Balzac ${ }^{44}$. Dans ce nouveau dispositif se retrouvent $a$ priori sur le même pied relations non tensives (à constante), relations tensives et relations "semi-tensives", c'est-à-dire non symétriques. En effet, les relations converse et inverse ne sont pas toujours symétriques relativement au type de variation (augmentation/ diminution) et/ou au facteur variant (élément $X$ ou élément $Y$ ). Il est ainsi possible de concevoir une relation qui soit converse de $\mathrm{X}$ à $\mathrm{Y}$, mais pas de $\mathrm{Y}$ à $\mathrm{X}$ : une augmentation de $\mathrm{X}$ entraîne celle de $\mathrm{Y}$, mais une augmentation de $\mathrm{Y}$ n'entraîne pas celle de $\mathrm{X}$ (ou entraîne sa diminution). Évidemment, les relations converse et inverse typiques sont symétriques ${ }^{45}$.

\subsection{Schéma tensif et courbe d'euphorie esthétique}

Les courbes d'euphorie esthétique, que nous avons présentées en détails ailleurs ${ }^{46}$, constituent (1) une représentation cognitive et

44. Zilberberg produit parfois des schémas tensifs qui placent sur un axe un type d'élément qui relève a priori de l'autre axe. Ainsi, définissant la vitesse et la lenteur comme relevant du tempo et donc de l'axe des intensités, Zilberberg (Éléments de sémiotique tensive, ouvr. cité) produit tout de même un schéma où la vitesse est sur l'axe des intensités et la lenteur sur l'axe des extensités. La lenteur est-elle nécessairement plus extense que la vitesse? S’agit-il encore d'un schéma tensif? Par ailleurs, le sémioticien fait aussi parfois correspondre les points minimal et maximal des axes tensifs, chacun renvoyant respectivement à l'absence (représentée par zéro) et à la présence (représentée par 1) de la variable placée sur l'intensité ou sur l'extensité. La même question se pose à nouveau: s'agit-il encore d'un schéma tensif?

45. Si corrélation il y a, elle ne sera pas nécessairement stricte: "Il n'est pas exigé de la corrélation stricte qu'elle soit stricte, puisqu'elle ne saurait l'être. En effet, la corrélation actualise une direction dont la réalisation rencontre des résistances, des obstructions et, par voie de conséquence, des retards » (Claude Zilberberg, Éléments de sémiotique tensive, ouvr. cité). Nous sommes tenté, par symétrie, d'ajouter que des effets anticipatoires peuvent également se faire jour.

46. Louis Hébert, "Typologies et segmentations du temps. Les courbes d'euphorie esthétique», dans Louis Hébert et Lucie Guillemette (dir.), Signes des temps. Temps et temporalités des signes, Québec, Presses de l'Université 
- leur nom l'indique assez - visuelle (2) des variations, (3) en fonction du temps, (4) de l'intensité des effets esthétiques euphoriques (5) générés (ou qui auraient pu, dû l'être) par une production sémiotique (ou sémiotisée: par exemple, un coucher de soleil comme spectacle) (6) dans l'une ou l'autre des instances de la réception (par exemple, le lecteur ou le spectateur empiriques $\left.{ }^{47}\right)$.

Les courbes d'euphorie esthétique permettent de rendre compte des variations d'intensité esthétique dans tout phénomène esthétique se déployant, ou déployé de force, dans le temps: productions sémiotiques dont la succession des unités à interpréter est fortement (par exemple, dans un texte écrit) ou absolument (par exemple, dans les arts du spectacle) contrainte; mais aussi productions sémiotiques réputées, à tort ou à raison, non ou peu temporelles (par exemple, un tableau), qu'on temporalise alors en y insérant ou reconnaissant une succession quelconque d'unités (par exemple, en organisant un parcours interprétatif sérialisant les figures d'un tableau).

Remarque: courbes esthétiques et autres courbes

Les mêmes courbes, départies de leur adjectif "esthétiques» ou imparties d'un autre, s'appliquent également à la description de phénomènes non esthétiques (du moins $a$ priori) qui impliquent (1) l'écoulement du temps, (2) la mesure d'un type d'intensité quelconque et (3) le phénomène processuel pour lesquels ils interviennent. On aura ainsi, par exemple, une courbe thymique (non esthétique), une courbe dramatique, une courbe orgasmique, celle d'une série de coups de marteau d'un artisan, etc.

En instaurant une segmentation triadique (intensités de l'euphorie basse, moyenne et élevée; positions temporelles initiale, médiane et finale) et en utilisant un temps irréversible, nous avons dégagé une typologie de 27 courbes esthétiques. Par exemple, si le Boléro de Ravel prévoit chez l'auditeur, et le réalise en effet, une ligne droite ascendante; d'autres productions ou parties de productions sémiotiques, qui ont les mêmes aspirations ne

Laval, 2005, p. 59-85. Nous atténuons ici sensiblement la certitude, que nous avions dans ce précédent ouvrage, du caractère non tensif des courbes d'euphorie esthétique.

47. Pour des détails sur les instances de la production et de la réception, voir Louis Hébert, Dispositifs pour l'analyse des textes et des images, ouvr. cité. 
suscitent chez le récepteur qu'une morne droite horizontale et ce, au plus bas niveau d'intensité...

Remarque: nature tensive des courbes d'euphorie

La question se pose: nos courbes d'euphorie esthétique relèvent-elles du schéma tensif? Le schéma tensif corrèle des éléments relevant de deux classes précises, d'une part, en ordonnée, des intensités et, d'autre part, en abscisse, des extensités, des étendues. Il appert que l'ordonnée de nos courbes correspond bien à ce qu'entend la sémiotique tensive par «intensités»: nous avons là non seulement une intensité, mais l'intensité d'un phénomène, l'axiologie, lui-même jugé typique de l'axe des intensités. Quant à notre abscisse, elle peut être ramenée à une forme d'extensité, plus précisément de nature temporelle. Cependant, il convient de distinguer le temps cumulatif et le temps successif, plus exactement le temps comme durée et le temps comme locatif (de même qu'en grammaire on distingue le cardinal et l'ordinal). Nos courbes reposent sur du temps locatif qui permet de définir les bornes d'une durée. Les courbes tensives peuvent évidemment reposer sur du temps considéré du point de vue de la durée, mais le peuvent-elles, dans l'esprit de leurs concepteurs, sur du temps locatif? S'agit-il encore d'étendue, d'extensité? Les courbes tensives, même lorsque le temps n'est pas en abscisse, ne se prêtent pas pour autant uniquement à des approches atemporelles. En effet, la typologie des quatre schémas tensifs repose sur un critère de succession des positions tensives (toute succession se produisant, en principe du moins, dans le temps). Les schémas tensifs - tout comme les déplacements tensifs - combinent alors trois variables : intensités, extensités et positions temporelles. Peut-on dire que nos courbes se limitent à deux variables ou faut-il considérer que la double orientation possible a priori n'a pas été retenue parce que non pertinente pour le type de substrat temporel considéré?

À tout le moins, notre typologie pourra servir de point de départ pour une typologie des schémas et déplacements tensifs dans une sectorisation triadique. Pour la compléter, il faudra ajouter trois «courbes» qui seraient des droites verticales (logées respectivement sur les extensités basse, moyenne et élevée) et tenir compte de la double orientation possible pour l'ensemble des courbes : par exemple, les courbes 7 et 8 sont identiques et 
simplement orientées temporellement de manière différente; les courbes 25,26 et 27, de mêmes que les trois droites verticales, connaissent deux orientations chacune. Cette typologie permet notamment de distinguer des schémas de l'ascendance qui se conforment à différentes courbes temporelles: 11, 15, 16, 23, etc. 
Typologie des courbes d'euphorie esthétique
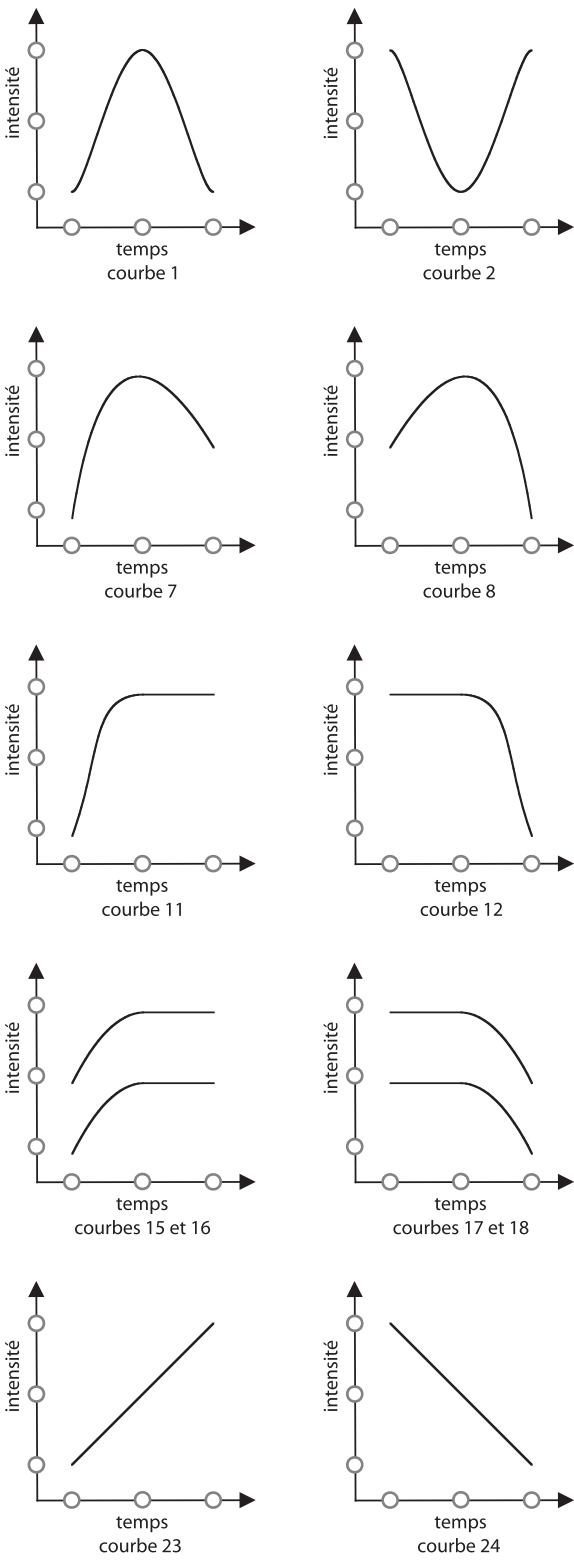

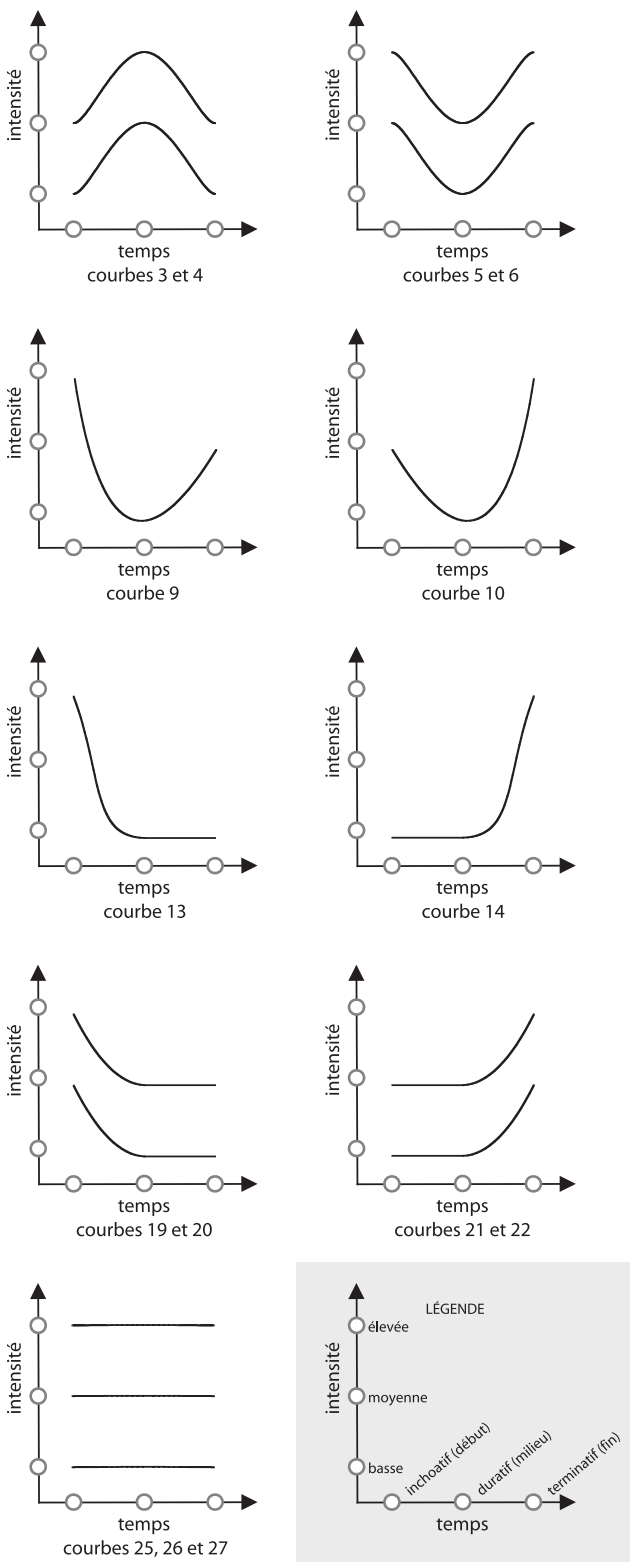


\section{Application : la connaissance selon Pascal}

Prenons cette pensée de Pascal sur la connaissance: «Puisqu'on ne peut être universel et savoir tout ce qui peut se savoir sur tout, il faut savoir peu de tout. Car il est bien plus beau de savoir quelque chose de tout que de savoir tout d'une chose; cette universalité est la plus belle. Si on pouvait avoir les deux, encore mieux, mais s'il faut choisir, il faut choisir celle-là, et le monde le sent et le fait, car le monde est un bon juge souvent ${ }^{48}$ ».

Le mot "tout", dans cette réflexion, n'est pas à prendre chaque fois au sens fort, absolu, mais parfois au sens de «beaucoup». En effet, l'homme, chez Pascal, est pris entre néant et infini, et ne peut de ce fait accéder ni à l'un ni à l'autre, comme le montre cette autre pensée ${ }^{49}$ :

Car enfin qu'est-ce que l'homme dans la nature? Un néant à l'égard de l'infini, un tout à l'égard du néant, un milieu entre rien et tout, infiniment éloigné de comprendre les extrêmes. La fin des choses et leurs principes sont pour lui invinciblement cachés dans un secret impénétrable.

Également incapable de voir le néant d'où il est tiré et l'infini où il est englouti, que fera-t-il donc $[\ldots]$ ?

[...] Ces extrémités [le néant et l'infini] se touchent et se réunissent à force de s'être éloignées, et se retrouvent en Dieu, et en Dieu seulement.

Connaissons donc notre portée. Nous sommes quelque chose et ne sommes pas tout. Ce que nous avons d'être nous dérobe la connaissance des premiers principes qui naissent du néant, et le peu que nous avons d'être nous cache la vue de l'infini.

$[\ldots]$

Voilà notre état véritable. C’est ce qui nous rend incapables de savoir certainement et d'ignorer absolument.

Si l'intensité s'applique à la profondeur de la connaissance et l'extensité à l'étendue du champ de cette connaissance, on situera ainsi quatre différents types de «connaisseurs» et de connaissances:

48. Blaise Pascal, Pensées, ouvr. cité, fragment 183, p. 607.

49. Blaise Pascal, Pensées, ouvr. cité, fragment 185, p. 610-612. 
Schéma tensif de la connaissance

\begin{tabular}{|l|l|l|}
\hline \multicolumn{1}{|c|}{ EXTENSITÉ } & \multicolumn{1}{|c|}{ BASSE } & \multicolumn{1}{c|}{ ÉLEVÉE } \\
INTENSITÉ & 2. Savoir beaucoup sur peu & $\begin{array}{l}\text { 4. Savoir beaucoup sur } \\
\text { beaucoup }\end{array}$ \\
\hline ÉLEVÉE & $\ldots \ldots \ldots \ldots \ldots \ldots \ldots \ldots \ldots \ldots$ \\
BASSE & 1. Savoir peu sur peu & 3. Savoir peu sur beaucoup \\
\hline
\end{tabular}

Pascal exploite les quatre zones et les hiérarchise: la meilleure zone, la quatrième, étant inaccessible aux hommes, il faut préférer la troisième à la deuxième; la première zone, implicitement présente, est dévalorisée: elle dépeint l'état initial de l'homme qu'il faut quitter («il faut savoir peu de tout»). Le connaisseur, situé à la jonction d'une extensité élevée et d'une intensité faible (mais tout de même supérieure à celle de l'homme ordinaire), correspond à l'idéal classique, auquel souscrit Pascal. En effet, à la différence de l'homme renaissant, dont l'idéal est celui du savoir universel (pensons au savantissime Pic de la Mirandole), l'«honnête homme» classique doit savoir, et ne peut que savoir, un peu de tout ${ }^{50}$. La conception classique relève d'un pessimisme cognitif; la conception humaniste, d'un optimisme cognitif où l'homme doit, en quelque sorte, rivaliser avec ce Dieu qui sait tout de tout.

Sur un autre plan, deux conceptions de la connaissance semblent se conjuguer chez Pascal. La première conception, qui se rapporte au potentiel cognitif de l'homme, postule une sorte d'énergie cognitive constante et instaure une corrélation de type inverse entre les deux axes: tout comme la pression d'un gaz diminue si on augmente le volume qu'il occupe, l'intensité de la connaissance décroît si le nombre d'objets qu'elle veut embrasser croît. Théoriquement possible, la corrélation converse, où l'énergie cognitive varierait, est implicitement jugée impossible dans la pratique. La seconde conception, qui se rapporte au capital des connaissances, postule une augmentation possible des connaissances par accroissement de l'une des deux valences à la

50. Jacques Fontanille (Sémiotique du discours, ouvr. cité, p. 186) considère qu'aujourd'hui l' "homme cultivé " est astreint à la même discipline: il est supposé "connaître un peu de tout, c'est-à-dire avoir des connaissances étendues mais sans se piquer de rien, sans se donner pour spécialiste». 
fois, soit de l'intensité, soit de l'extensité (la déperdition des connaissances n'est pas envisagée).

Or, aucun des schémas tensifs élémentaires ne prévoit que l'une des valences soit constante tandis que l'autre varie. On pourrait arguer que le tracé qui va de la zone un à la zone trois est en pente et que le peu de la seconde est supérieur au peu de la première; bref, que l'apparente horizontale se révèle être une diagonale si l'on augmente la précision de l'analyse en tenant compte des multiples positions possibles dans une même zone. Quoi qu'il en soit, il est plus difficile d'appliquer cette même objection au tracé qui va de la zone un à la zone deux: l'approfondissement de la connaissance peut ne pas entraîner une augmentation de l'extensité, s'il s'applique à un objet de savoir déjà inclus dans le savoir peu initial. Nous revenons donc à notre point de départ: comment intégrer la description de ces droites orthogonales dans le schéma tensif? Ne faisons état que de l'une des solutions envisageables et distinguons - ici comme dans d'autres descriptions tensives, voire dans toutes - les schémas tensifs élémentaires, nécessairement marqués par une relation converse ou inverse, des déplacements tensifs élémentaires, qui peuvent ne pas connaître une telle relation. Pascal n'utilise que deux des déplacements non converses et non inverses, sans prévoir en particulier le passage de la zone deux à la zone un et de la zone trois à la zone un.

Nous avons préféré ne pas indiquer de lexicalisations, de noms pour les zones, dans notre tableau. Dans la plupart des schémas tensifs, la lexicalisation des zones pose problème: d'une part, il n'existe pas toujours une lexicalisation valable pour une zone donnée et, d'autre part, plusieurs lexicalisations et classements peuvent convenir à une même zone, notamment en raison de la récursivité des relations tensives.

Par exemple, si l'on prend la paire ignare/homme ordinaire, le premier terme relèvera de la première zone et le second, de la troisième; même chose pour la paire homme ordinaire/honnête homme, le premier terme relevant de la première zone et le second, de la troisième. Qu'arrive-t-il si l'on veut rendre compte de la totalité de la triade? Si l'on opte toujours pour un schéma à quatre zones, on classera deux éléments dans une même zone, mais dans des positions légèrement différentes sur l'un et/ou l'autre des deux axes, et le troisième élément logera seul dans sa zone. Ainsi, on aura: ignare et homme ordinaire dans la première 
zone, honnête homme dans la troisième, ou ignare dans la première et homme ordinaire et honnête homme dans la troisième.

Prenons maintenant un schéma à plus de quatre zones. Prévoyons, pour les deux axes, des secteurs de force basse, moyenne et élevée. L'ignare se situe alors à la jonction de l'intensité et de l'extensité basses; l'homme renaissant, à la jonction de l'intensité et de l'extensité élevées. Quant aux autres figures de connaisseur que nous avons dégagées dans le schéma précédent, si plusieurs classements concurrents en sont possibles, ceux que nous proposons sont les suivants: l'honnête homme pourra être placé à la jonction de l'intensité moyenne et de l'extensité élevée, l'homme ordinaire, à la jonction de l'intensité et de l'extensité moyennes; le spécialiste, à la jonction de l'intensité élevée et de l'extensité moyenne. Le principe qui nous guide dans le classement de ces trois dernières figures de connaisseur est le suivant: il serait injuste pour le spécialiste de le situer dans une extensité plus faible que celle de l'homme ordinaire et, pour l'honnête homme, de lui prêter une intensité de connaissance plus faible que celle de l'homme ordinaire.

Il reste alors quatre des neuf zones de ce schéma pour classer, éventuellement, d'autres types de connaisseur et de connaissance. Le nombre de zones pertinentes dépend de l'ensemble à décrire. Par exemple, pour décrire l'ensemble des types de médecin, deux des zones définies par une segmentation dyadique sont suffisantes: le spécialiste ${ }^{51}$ (ou médecin spécialiste) doit être reporté dans la zone deux et l'omnipraticien (ou médecin généraliste) dans la zone trois. Cette interdéfinition ne prend pas en compte les savoirs non médicaux et exclut le non-médecin : si l'omnipraticien en sait "peu» sur chaque secteur de la médecine, c'est relativement au spécialiste et non à l'homme de la rue, qui en sait "très peu».

Une autre pensée de Pascal portant sur la connaissance vient complexifier l'analyse ${ }^{52}$ :

Le monde juge bien des choses, car il est dans l'ignorance naturelle qui est le vrai siège de l'homme. Les sciences ont deux extrémités qui se touchent, la première est la pure ignorance

51. L'interniste est à concevoir comme un spécialiste du résiduel plutôt que comme un «multispécialiste»: il traite, par définition, des maladies ne relevant pas d'une spécialité précise.

52. Blaise Pascal, Pensées, ouvr. cité, fragment 77, p. 566. 
naturelle où se trouvent tous les hommes en naissant, l'autre extrémité est celle où arrivent les grandes âmes qui, ayant parcouru tout ce que les hommes peuvent savoir, trouvent qu'ils ne savent rien et se rencontrent en cette même ignorance d'où ils étaient partis, mais c'est une ignorance savante qui se connaît. Ceux d'entre deux, qui sont sortis de l'ignorance naturelle et n'ont pu arriver à l'autre, ont quelque teinture de sotte science suffisante et font les entendus. Ceux-là troublent le monde, et jugent mal de tout.

Le peuple et les habiles composent le train du monde; ceux-là le méprisent et sont méprisés. Ils jugent mal de toutes choses, et le monde en juge bien.

Dans cette troisième pensée, à la différence de ce qui se passe dans la première pensée, la zone un est valorisée, du moins cette portion de la zone que nous situerons dans le coin inférieur gauche et qui correspond à l'ignorance naturelle absolue où se trouve l'homme à la naissance. On remarque, comme dans la seconde pensée, une valorisation des extrêmes et une dévalorisation de l'intermédiaire, position du demi-savant qui, ayant quitté irrémédiablement l'ignorance naturelle, n'a pas encore atteint, ne pourra jamais atteindre, la docte ignorance ${ }^{53}$.

Considérons que le passage du grand savoir à l'ignorance naturelle est catégoriel plutôt que graduel ${ }^{54}$ (puisque l'on passe sans transition de l'une à l'autre) et que la connaissance qui, un degré plus élevé, mène à l'ignorance naturelle, se trouve dans la zone trois, dans sa partie supérieure droite. Reste à savoir où loge la docte ignorance. Comme son nom l'indique, la docte ignorance n'est pas un retour à l'ignorance naturelle: elle ne dessine pas un cercle mais une spirale. Ce qui la distingue de l'ignorance, en sus d'un savoir supérieur, est ce savoir paradoxal qui se sait ignorer. Pascal accorde beaucoup d'importance à la conscience réflexive, à un «savoir-que» qui implique le sujet pensant lui-même. Il écrit: «L'homme n'est qu'un roseau, le plus faible de la nature, mais c'est un roseau pensant. Il ne faut pas que l'univers entier s'arme pour l'écraser; une vapeur, une goutte d'eau suffit pour le tuer. Mais quand l'univers l'écraserait, l'homme serait encore plus noble que

53. Ailleurs, Pascal (Pensées, ouvr. cité, fragment 468, p. 745) valorise la position intermédiaire: "C'est sortir de l'humanité que de sortir du milieu.// La grandeur de l'âme humaine consiste à savoir s'y tenir [...]. »

54. Si le passage du grand savoir à l'ignorance savante est graduel, il faut se demander quand (c'est-à-dire où, sur le plan) il commence, s'il se produit par avancées égales ou en s'accélérant, et quand il est complété. 
ce qui le tue puisqu'il sait qu'il meurt et l'avantage que l'univers a sur lui, l'univers n'en sait rien. ${ }^{55}$ " En paraphrasant Pascal, on dira que l'homme est grand parce qu'il sait (ou peut savoir) qu'il ignore.

Une des réponses possibles à notre interrogation consiste à considérer que le passage du grand savoir à la docte ignorance réside non pas dans un saut de la zone trois à la zone un, mais dans la transformation même du plan où le connaisseur a conscience de se situer: les axes, au lieu de demeurer stables, s'allongent (s'allongent-ils comme un horizon qui s'éloigne continûment, mais régulièrement, ou comme un horizon qui s'éloignerait de plus en plus?), de telle sorte que le connaisseur se trouve proportionnellement dans la situation de l'ignorant naturel. L'ignorant savant a alors véritablement pris la mesure de ce qu'il ignore. Cela nous rappelle que tout positionnement est déterminé par l'appréciation d'un observateur, de sorte que l'ignorant naturel croit se situer à la jonction d'une intensité et d'une extensité plus élevées qu'en réalité, puisqu'il ignore jusqu'à l'étendue de ce qu'il pourrait connaître mais, en fait, ignore...

55. Blaise Pascal, Pensées, ouvr. cité, fragment 186, p. 614. 